\title{
RELATIONS Vs FUNCTIONS
}

\section{Prof .Sunitha Janga \\ Assistant Professor, T. John Institute of Management and Science, Bangalore, India, Email Id:sunithaj@tjohngroup.com}

\begin{abstract}
This paper aims to understand the concepts of relations and functions as well with the inclusive of ordered pairs ,Cartesian product, domain ,codomain ,range of a function. This paper also focusing on some of the special types of functions and also facilitates better idea on when a relation can be a function. This article also provides clear picture on what magic involved in the given relation to identify whether a function using graphing functions.
\end{abstract}

Keywords: Cartesian Product, Codomain, Domain, Functions, Graphing functions, Ordered Pairs, Range, Relations .

\section{INTRODUCTION}

\section{Ordered Pairs:}

For any two given sets $\mathrm{A}$ and $\mathrm{B}$,An element of the set $\mathrm{A}$ and An element of the set B separated by comma enclosed in parenthesis is called “Än ordered pair".

For example, $(1,2)$ is an ordered-pair number; the order is designated by the first element 1 and the second element 2 . The pair $(2,1)$ is not the same as $(1,2)$ because of the different ordering. These ordered pairs are represented as Relations or Functions.

Cartesian Product: If $\mathrm{X}$ and $\mathrm{Y}$ are two sets, then the Cartesian product of $\mathrm{X}$ and $\mathrm{Y}$ is defined as

$$
\mathrm{X} * \mathrm{Y}=\{(\mathrm{x}, \mathrm{y}):(\mathrm{x} \in \mathrm{X}) \text { and }(\mathrm{y} \in \mathrm{Y})\}
$$

Cartesian product of 2 sets is set of all possible ordered pairs of two given sets. In every ordered pair ,First element is belongs to the first set, Second element belongs to the second set.

\section{Example (Cartesian product)}

If $\mathrm{A}=\{1,2,3\}$ and $\mathrm{B}=\{\mathrm{a}, \mathrm{b}, \mathrm{c}, \mathrm{d}\}$,

then $A \times B=\{(1, a),(1, b)(1, c),(1, d),(2, a),(2, b),(2, c)$, $(2, d),(3, a),(3, b),(3, c),(3, d)\}$..

\section{Relation:}

A relation $\mathrm{R}: \mathrm{A} \rightarrow \mathrm{B}$ (Where $\mathrm{A}$ and $\mathrm{B}$ are non empty sets) is defined as sub set of $\mathrm{A} \times \mathrm{B}$.

A relation organizes ordered pairs into a set.

$$
\mathrm{R} \subseteq \mathrm{A} \times \mathrm{B}
$$

The set of first components of ordered pair is called" the domain", The set of second components is called "the Codomain"

For example,

Let's Say $A=\{a, b\}, B=\{$ yellow, white,pink $\}$ then :
$\mathrm{R}=\{(\mathrm{a}, \mathrm{yellow})\},(\mathrm{b}$, white $),(\mathrm{a}$, pink $)\}$ is a subset of $\mathrm{A} \times \mathrm{B}$ therefore $\mathrm{R}$ is a relation

Example: Suppose Ages of four men is listed below.

\begin{tabular}{|l|c|c|c|c|}
\hline $\begin{array}{l}\text { Name of the } \\
\text { Person }\end{array}$ & Madhu & Bobby & Ravi & Ramesh \\
\hline Person's Age & 40 & 50 & 35 & 45 \\
\hline
\end{tabular}

The pairing of Person Name and Their ages is formed as a relation and can be written as a set of ordered-pair numbers. $W=\{($ Madhu, 40), (Bobby, 50), (Ravi, 35), (Ramesh,45) $\}$

Domain, inputs, codomain, range of a function:

If $\mathrm{f}: \mathrm{A} \rightarrow \mathrm{B}$ is a function,then

- The set A is called the domain, and the elements of A are called inputs to $\mathrm{f}$ (so the domain is the first elements where the inputs live).

- The set B is called the codomain.

- The set of the elements of B that are assigned to some element in $\mathrm{A}$

The range of $f$ is the set $f(A)=\{b: b \in B$ and $b=f(a)$ for some $a \in A\}$

\section{FUNCTIONS}

A Relation ' $\mathrm{f}$ ' from set A to set $\mathrm{B}$ is said to be a function if every element of set $\mathrm{A}$ has one and only one image in set B.

Let us examine the following Relations are Functions?

$$
R=\{(2,1),(3,1),(4,2)\}
$$

Since 2,3,4 are the elements of the domain of $\mathrm{R}$ having their unique images, $\mathrm{R}$ is a function

ii) $\mathrm{R}=\{(1,2),(2,3),(3,4),(4,5),(5,6),(6,7)\}$ 
Same as above, all the elements of domain of $\mathrm{R}$ have one and only one image. So it is a function

iii)

$$
\mathrm{R}=\{(2,2),(2,4),(3,3), 4,4)\}
$$

$\mathrm{R}$ is not a function as element first element 2 corresponds to two images $2 \& 4$

Some more Examples: Determine whether the following are functions
a) $X=\{(1,3),(0,3),(2,1),(4,2)\}$
b) $Y=\{(1,6),(2,5),(1,9),(4,3)\}$

\section{Solution:}

a) $X=\{(1,3),(0,3),(2,1),(4,2)\}$ is a function because all the first elements are different. (The second element does not need to be unique)

b) $C=\{(1,6),(2,5),(1,9),(4,3)\}$ is not a function because the first element, 1 , is repeated

Here are some examples:
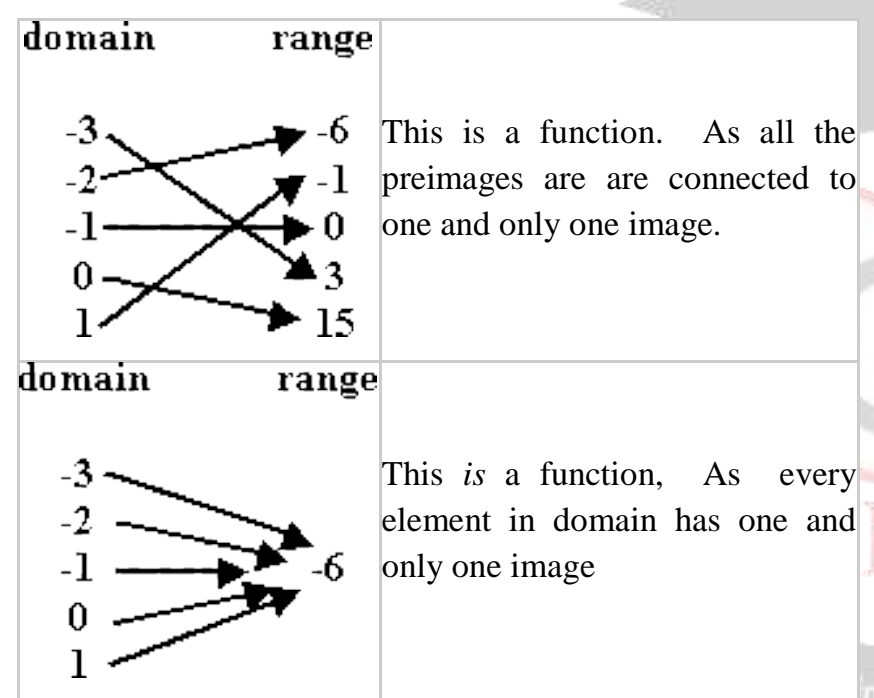

Note:A function associates each element in its domain wirh one and only one element in its Range

\section{Special Functions}

Special functions and their equations have recognizable characteristics.

\section{Constant Function:}

$f(x)=c$

Here $c$ can be any real number.

Graph of this function is shown as a horizontal line as follows

Example:. Here is the graph of $f(x)=4$

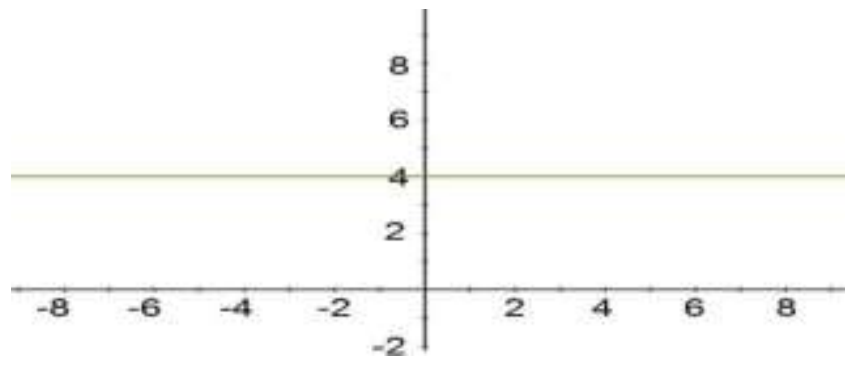

\section{Identity Function:}

$f(x)=x$

For the identity function, the $\mathrm{x}$-value is the same as the $\mathrm{y}$ value. The graph is a diagonal line going through the origin.

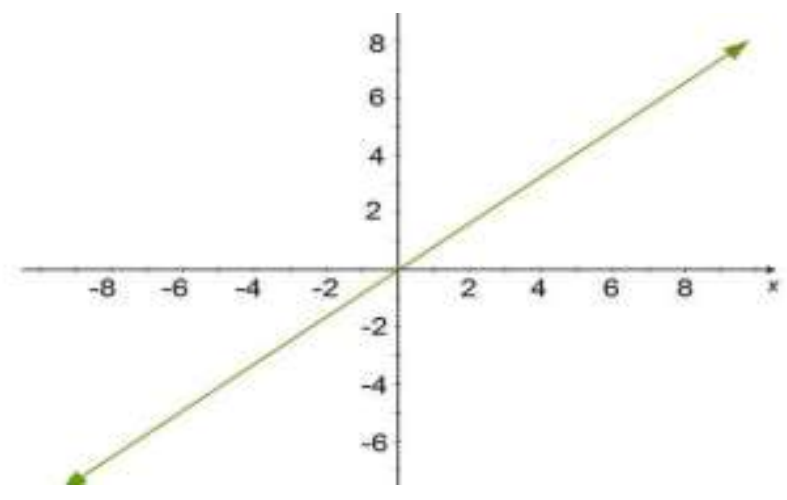

\section{Linear Function:}

$f(x)=m x+b$

An equation written in the slope-intercept form is the equation of a linear function, and the graph of the function is a straight line.

Here is the graph of $f(x)=3 x+4$

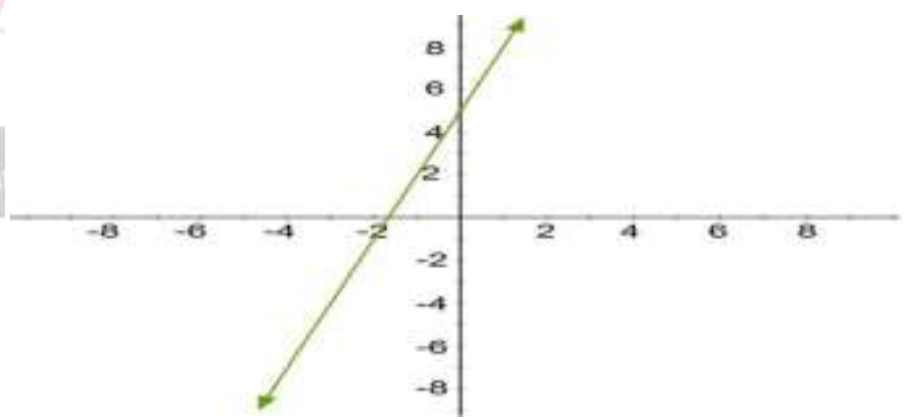

Modulus Function:

$f(x)=|x|$

$$
\begin{aligned}
& f(x)=x, \text { if } x>0(\text { for all non negative values of } x) \\
&=-x, \text { if } x<0 \text { (for all negative values of } x) \\
&=0, \text { if } x=0
\end{aligned}
$$


This function graph is easy to recognize with its V-shaped Graph as shown below

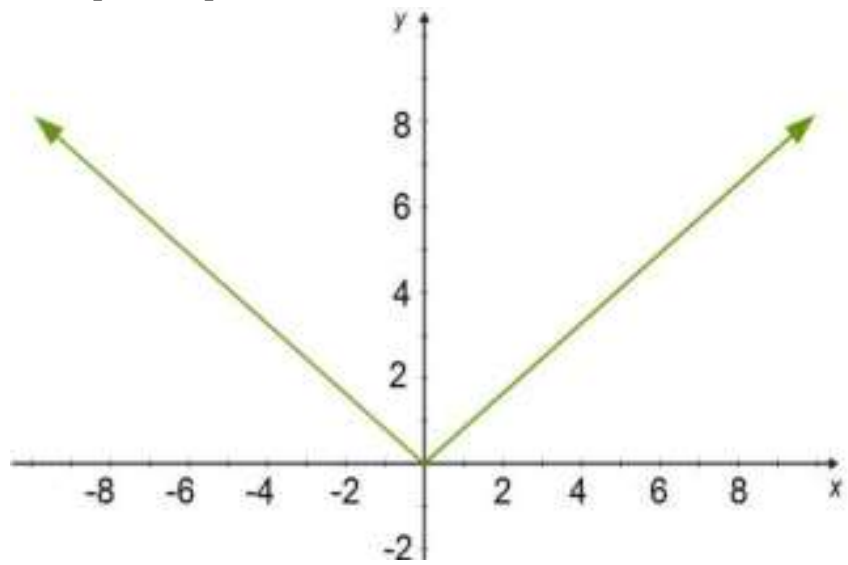

This is just a sample of the most common special functions.

\section{GRAPHING FUNCTIONS}

Using inputs and outputs listed in tables, maps, and lists, makes it is easy to plot points on a coordinate grid. Using a graph of the data points, you can determine if a relation is a function by using the vertical line test.

This test provides us very easier way to check when a Relation can be a Function.

Take a look at the graph of this relation map. If you were to draw a vertical line through each of the points on the graph, each line would touch at only one point, so this relation is a function.

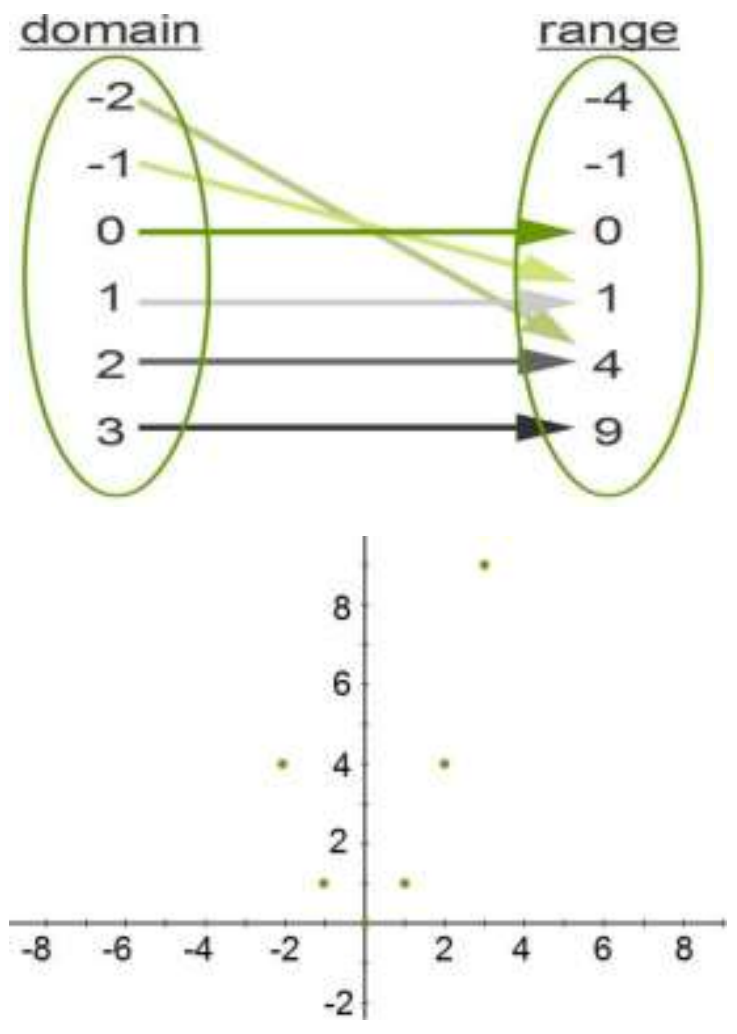

"Vertical Line Test":

Let's examine this relation given is a function which is also shown graphically below:
Let's say Relation $\mathrm{R}=\{(2,3),(2,-2)\}$

We know that this is not a function since first element 2 corresponds to $3 \&-2$

In the graph ,The Relation looks like this:

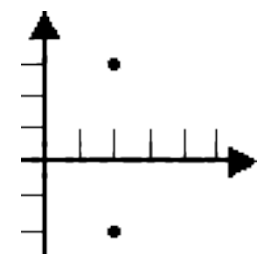

Observe that A vertical line can be drawn through the two given points, like this:

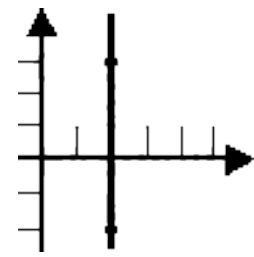

This is how A function can be identified from the graph .

If any vertical line which is drawn through the graph cuts the graph at more than one point, then the relation is not a function. This is known as "Vertical Line Test"

\section{Inverse Functions}

The inverse function reverses elements of domain with its elements of Range

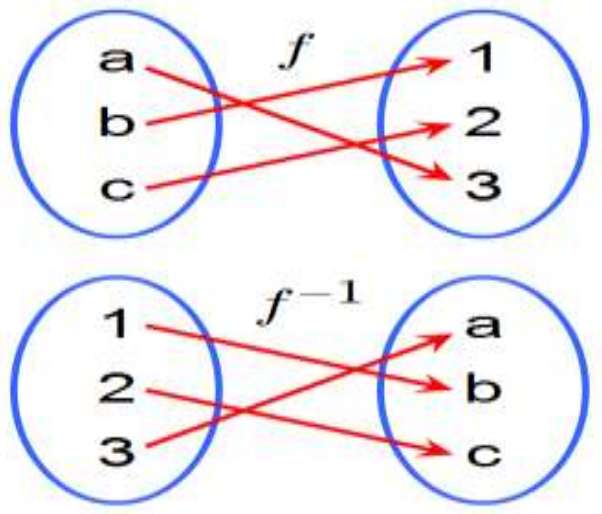

Suppose: Let $f(x)=3 x-4$

The inverse of $f(x)=3 x-4$ is $f-1(x)=(X+4) / 3$

Note: Every inverse function of a function may not be a function.We can also use Vertical line test to prove.

\section{Function Operations:}

Addition, subtraction, multiplication and division of functions.

- $\quad f(x)+g(x)=(f+g)(x)$

- $\quad \mathrm{f}(\mathrm{x})-\mathrm{g}(\mathrm{x})=(\mathrm{f}-\mathrm{g})(\mathrm{x})$

- $f(x) \times g(x)=(f \times g)(x)$

- $f(x) g(x)=f g(x)$

Let us look at two examples of function operations:

If $f(x)=x^{2}+x+2, g(x)=x+5$ then 
- $(f+g)(x)=x^{2}+x+2+x+5=x^{2}+2 x+7$

- $(f-g)(x)=x^{2}+x+2-x-5=x^{2}-3$

What is the product of these two functions? Simply multiply the expressions.

- If $\mathrm{f}(\mathrm{x})=\mathrm{x}+4, \mathrm{~g}(\mathrm{x})=\mathrm{x}+7$ then

- $(\mathrm{f} \times \mathrm{g})(\mathrm{x})=(\mathrm{x}+4) \times(\mathrm{x}+7)=\mathrm{x}^{2}+11 \mathrm{x}+28$

Important Note:All functions are relations, but all relations are not functions

Case-I: All functions are relations

A function is a particular kind of relation between sets. A function takes every element $\mathrm{x}$ in a starting set, called the domain, and tells us how to assign it to exactly one element $\mathrm{y}$ in an ending set, called the range.

For example, each person is in the following table is paired with a number representing his or her height:

$\mathrm{SRIDHAR} \rightarrow 185, \mathrm{RAMESH} \rightarrow 167, \mathrm{SAMPATH} \rightarrow 184$, $\mathrm{RAVI} \rightarrow 158$

The given relation $\{($ Sridhar,185), (Ramesh , 167),

(Sampath ,184), (Ravi, 158) $\}$ is a function as every person is pairs with exactly one number, their height. The domain is (Sridhar, Ramesh, Sampath, Ravi). The range is (185, $167,184,158)$.

By this we conclude one thing says All functions are relations but all relations are not functions

For instance, matching a person's age with their height does not give a function: Say Ramesh and Ravi are both 15. In this case, 15 would get paired with both 167 and 158, meaning that every age is not paired with exactly one height.

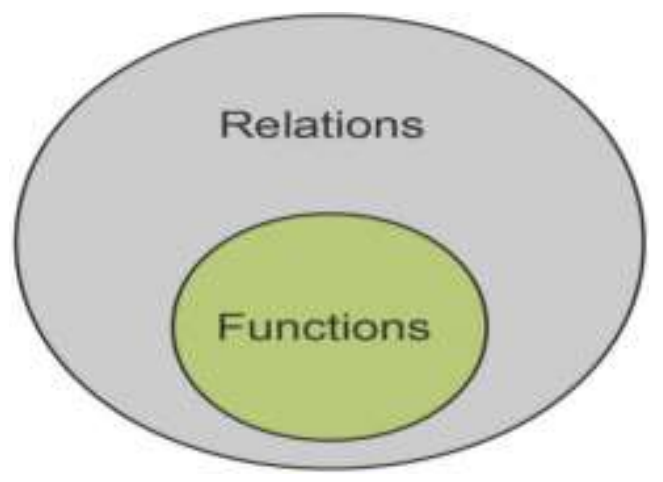

A function is a relation that for each element in the domain, there is only one image.

Here are some mappings of functions:

\section{domain}

range

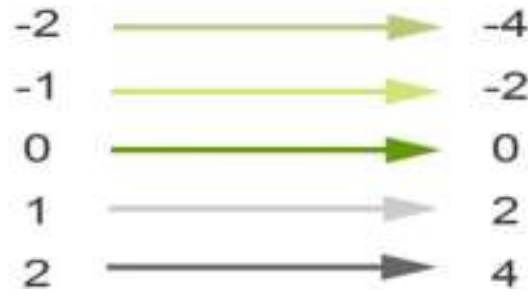

1.

domain

\section{range}

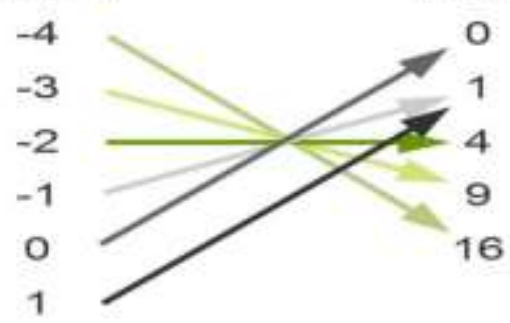

Although -1 and 1 have the same image, this relation is still a function because each preimage has just one image.

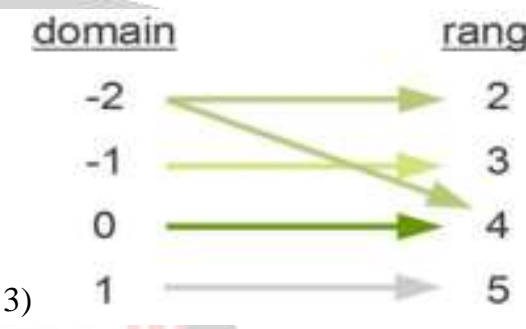

This mapping is not a function. -2 has more than one image.

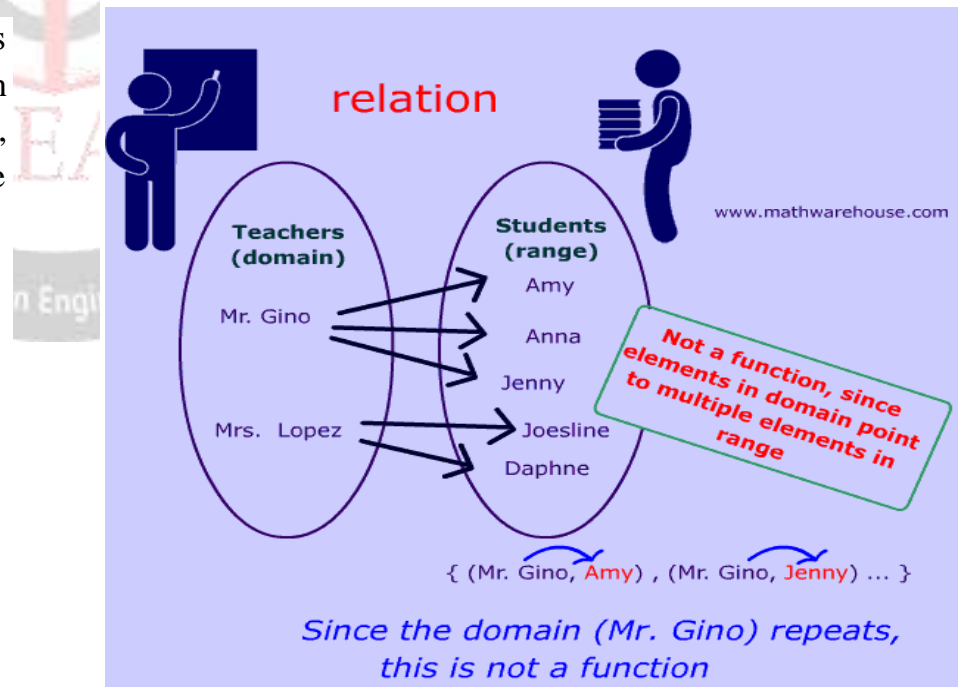

Example: Every Relation is not a function.

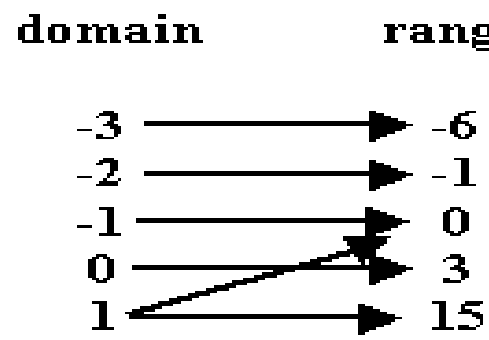


Observed that Above mapping is not a function as Element 1 in the domain has 2 images $0 \& 15$. but this is the relation

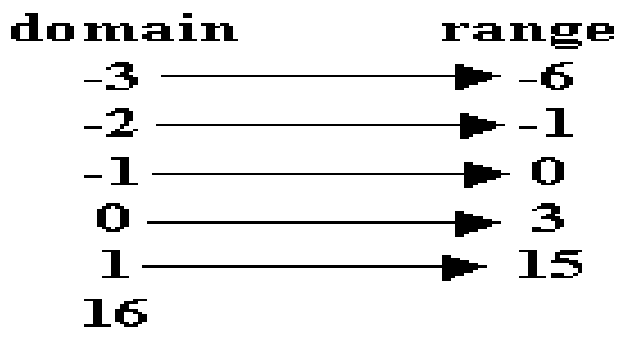

Each element of the domain that has a pair in the range is nicely well-behaved. 16 is in the domain, but it has no image.So this is not a function.

\section{CONCLUSION}

This paper was successful in explaining the concepts of relations and functions also facilitated on various ideas a relation can be a function. It provided a clear path in the given relation to identify a function graphically and in establishing basic operations on given functions mathematically.

\section{REFERENCES}

[1] ISC Mathematics,S,Chand Publications(An ISO 9001:2008 Company),2015

[2] https://sciencing.com/what-makes-a-relation-afunction-13712220.html

[3] https://www.onlinemathlearning.com/relationfunction.html

[4] http://www.math.uvic.ca/faculty/gmacgill/guide

[5] https://www.purplemath.com/

[6] https://www.researchgate.net/

[7] https://www.coloradotech.edu/

[8] https://danaernst.com/

[9] https://www.scribd.com/

[10] https://slidelegend.com/ 